\title{
Equivalencia clínica entre medicamentos genéricos y originales de marca utilizados en Enfermedades Cardiovasculares
}

Clinical equivalence between generic and brand name drugs used in cardiovascular disease

Aaron S y col. JAMA. 2008;300(21):2514-2526

\begin{abstract}
Objetivo
Analizar trabajos que hubieran comparado la equivalencia clínica entre medicamentos genéricos y originales de marca para el tratamiento de enfermedades cardiovasculares (ECV) y evaluar las perspectivas de los comentarios editoriales sobre el tema.

\section{Fuente y extracción de Datos}

Registros de publicaciones en MEDLINE, EMBASE y en Internacional Pharmaceutical Abstract desde 1984 hasta 2008, usando tres dominios de búsqueda: tipo de estudio, productos de interés (brand-name ú originales de marca, producto innovador, genéricos, patentes, etc.) y términos relacionados con ECV. La calidad metodológica fue evaluada mediante escala de Jadad para los ensayos clínicos aleatorizados (ECA) y la de nueve estrellas de Newcastle-Otawa para los estudios observacionales. Los autores subdividieron los fármacos como de Amplio Índice Terapéutico* (AIT) y como de Estrecho Índice Terapéutico* (EIT). El metanálisis se realizó obteniendo el coeficiente Cohen D (calculando las medias y los desvíos estándar) de los estudios incluídos y analizando el tamaño de efecto global, definiéndose a este último como muy pequeño si era menor a 0,2 ; pequeño $(0,2$ a 0,5$)$; mediano $(0,5$ a 0,8$)$ y grande cuando superaba 0,8 .
\end{abstract}

\section{Selección de estudios}

Fueron incluidos aquellos trabajos en inglés que hubieran comparado drogas cardiovasculares genéricas (aprobadas por FDA) con marcas originales patentadas, debiendo incluir como mínimo algún punto final de eficacia clínica y seguridad: signo vital, parámetro de laboratorio (p. ej: RIN) morbi-mortalidad ó alguna tasa de utilización del Sistema de Salud. Los editoriales fueron categorizadas de acuerdo a su postura acerca del reemplazo de medicamentos originales por genéricos: positiva, neutra o negativa.

\section{Resultados Principales}

De los 8556 artículos encontrados, fueron seleccionados 47 estudios (38 ECCA). Se obtuvo equivalencia clínica en los siguientes ECA de AIT: $7 / 7$ de $\beta$-bloqueantes, 10/11 de diuréticos, $5 / 7$ de bloqueantes cálcicos, $3 / 3$ de agentes antiplaquetarios, 2/2 de estatinas, 1/1 de inhibidores de la Enzima Convertidora de Angiotensina y $1 / 1$ de a-bloqueantes. La equivalencia clínica en drogas con EIT fue 1/1 en ECA de antiarrítmicos clase 1 (Propafenona) y $5 / 5$ en ECA de Warfarina.

El tamaño del efecto global $(n=837)$ fue de $-0,03$ (IC95\% $-0,15$ a 0,08 indicando la ausencia de evidencia de superioridad de las marcas originales respecto a los medicamentos genéricos. Sin embargo, de 43 editoriales, 23 expresaron un punto de vista negativo sobre sustituir marcas originales por drogas genéricas.

\section{Conclusiones}

A pesar de no encontrarse evidencia clínica de la superioridad del uso de medicamentos originales respecto a genéricos en ECV, muchas editoriales no recomiendan intercambiarlos.

Palabras claves: revisión sistemática, equivalencia clínica, medicamento genérico, originales de marca, enfermedades cardiovasculares.

Key words: systematic review, clinic equivalence, generic drug, original brand name, cardiovascular diseases.

Fuente de financiamiento: Attorney General Prescriber and Consumer Education Grant Program. El Dr.Kesselheim recibió el apoyo del Harvard School of Public Health.

\section{Comentario}

Se destaca de esta revisión sistemática el currículum de los autores, y su calidad metodológica. Como puntos débiles destacamos la utilización de un número reducido de individuos en comparación con la población real de enfermos cardiovasculares, el haber incluido una población principalmente sana y joven, gran cantidad de ensayos de bioequivalencia y no tanto clínicos y la limitación al idioma inglés en la selección de estudios. En la práctica diaria existe preocupación en los pacientes y en los profesionales de la salud al reemplazar medicamentos de marca por genéricos. El trabajo de Kesselheim y col. busca evidenciar la equivalencia clínica intentando dejar de lado las preocupaciones subjetivas, los ensayos no clínicos y los intereses particulares del circuito "Industria Farmacéutica-MédicoFarmacia". Debemos contextualizar las conclusiones a las que llegaron los autores ya que la equivalencia clínica obtenida se da en un marco regulatorio distinto al de nuestro país, con drogas aprobadas por la Agencia Regulatoria Estadounidense y exigencia de ensayos de bioequivalencia entre los medicamentos genéricos y los innovadores de marca. Sin embargo, creemos que este tipo de trabajo es el camino para aportar evi- dencia científica y objetividad a la hora de la implementación de una política de medicamentos acorde a nuestra situación económica, asegurando la accesibilidad, como la calidad y eficacia clínica de los medicamentos.

\section{Conclusiones de los comentadores}

Desde la aparición de la Ley 16.462 de Medicamentos (Ley Oñativia-1964) se ha hecho visible el conflicto de intereses y la presión de la industria farmacéutica sobre la economía argentina. Más cercano en el tiempo, la implementación de la Ley 25.649 de Prescripción de Medicamentos por nombre Genérico, profundizó el debate farmacoeconómico y el relativo al intercambio de marcas y eficacia clínica.

Mediante la contención del gasto en medicamentos se contribuye a la equidad y se mejora la accesibilidad a los tratamientos. Por otra parte, las autoridades sanitarias deberían garantizar la efectividad clínica mediante controles continuos a través de la utilización de Sistemas de Farmacovigilancia e incentivando el desarrollo y la inversión para obtener genéricos bioequivalentes'

Leonardo Garfi [ Coordinador de Fármacos e Insumos - Plan de Salud del Hospital Italiano de Buenos Aires leonardo.garfi@hospitalitaliano.org.ar ]

Ver glosario*

Garfi L, Schember M y Vidal G. Equivalencia clínica entre medicamentos genéricos y originales de marca utilizados en Enfermedades Cardiovasculares. Evid Act Práct Ambul Vol 13(1) 18. Ene-Mar 2010. Comentado de: Kesselheim A y col. Clinical equivalence of generic and brand-name drugs used in cardiovascular disease: a systematic review and meta-analysis. JAMA.2008 Dec 3;300(21):2514-26. Review. PMID: 19050195. 